\title{
Effective Early Stage Detection of COVID-19 Using Deep Learning
}

\author{
Swetha .M $\mathrm{M}^{\mathrm{a}, 1}$ and Rajendiran. $\mathrm{M}^{\mathrm{b}}$ \\ a PG Scholar, Dept of CSE, Panimalar Engineering College, Chennai, India \\ ${ }^{b}$ Professor, Dept of CSE, Panimalar Engineering College, Chennai, India
}

\begin{abstract}
The Corona virus Disease 2019 (COVID-19), which was formerly called as 2019 Novel Corona Virus[1] is a breath taking disease. It had its impact on millions of lives across the world. At present, as of March 2021, the rate of infection has declined throughout different parts of the world [2]. But it has been warned by scientists that this deadly disease can have its second wave over a period of time. Also, there is a possibility of this covid-19 to become a seasonal disease [3]. In such case, premature diagnosis of this virus is essential in order to save many lives. A kit called RT- PCR has been employed to detect the presence of this virus [4]. However, this method of prognosis takes time depending on the locality of the infected person [5]. This leads to the proliferation of the infection. Hence, an alternate procedure should be unearthed, which diagnose this disease within a short span of time. In this paper, a Deep Learning concept has been proposed which aids the timely detection of the corona virus infection. This, inturn reduces the spreading rate of the infection and decreases mortality rate.
\end{abstract}

Key words: Covid-19, Diagnosis, RT-PCR, Deep Learning.

\section{Introduction}

The Novel Corona virus has its origin in the city of Wuhan, china [6], in December 2019. It is now rooted in different countries over globe and has been proclaimed as a pandemic. As of March 2021, 116 Million patients has been infected by the corona virus across the world, out of which 91 Million patients has been recovered from it and 2 million patients had lost their lives [7]. The cause of this infection is a SARS-Cov-2 virus, which has changing or mutating nature. This dynamic character of the virus makes it difficult for the researchers to find a vaccine for the infection. However, many countries like India found a vaccination for covid-19 after a long fight and it has been employed for public use [8]. It is believed that this vaccine has it efficiency of around $85 \%$ [9]. And there is no assurance that the vaccinated person will never get the infection again. The only way to decrease the size of infection is by diagnosing it early. Hence, we are in need of a sophisticated method for diagnosing the disease. Here comes the concept of Deep Learning, which enlighten the scientists for developing a new procedure for the diagnosis.

\footnotetext{
${ }^{1}$ Swetha. M, PG Scholor, Dept of CSE, Panimalar Engineering College, Chennai;

E-mail: swetha@gmail.com
} 
A substantial research has been done by many researchers on the usage of Deep Learning and Machine Learning in covid-19 detection [10]. Here, a Deep Learning concept which involves convolutional neural network has been put forth . The CNN is trained and tested with available covid datasets. When the input CT scan of the suspected patient is given to the $\mathrm{CNN}$, it categorizes the data and detects the presence of infection. Once the presence of the virus is detected, the region which is affected by the virus can also be segmented by the network. This process can be done within a less amount of time with high performance.

\section{Related Work}

For the examination of the infection, many antigen kit has been employed around the world. But, it has been revealed that this tool has certain amount of negativity rate. To support this fact, an analysis has been made by analysts [11], which results in declaring the low performance of the procedure. In this experiment, 148 swabs were used, which gives of the sensitivity of $30.2 \%$. This shallow sensitivity proves that the antigen kit cannot be used as a main testing component.

In order to overcome the drawbacks of the toolkit diagnosis, many technical methods has been projected by different experimenters. These methods use CT scan of the patients, $\mathrm{X}$ - ray and ultrasounds of the lungs as input and diagnose the contamination. A review has been made which includes the use of ultrasound scan images of the lung as input [12] and gives the result. Here, it has been stated that the LUS method can be used for more accurate detection of pneumonia. Since it is of low cost, it can be performed anywhere, it can also be employed in the diagnosis of covid19.

Another method, which involves the use of CXR for the identification of disease had been described by Rodolfo M. Pereira [13]. Even though, the use of CT scan is accepted worldwide for easy diagnosis of any disease, CXR can also be used for diagnosing many disease, particularly in case of covid-19, since it incurs less cost and can be processed with more speed. Here, it is employed to identify the pneumonia which is affected as a result of corona virus infection. This method is best suitable for unbalanced environment, which works by extracting the texture feature of the CXR.

Shayan Hassantabar[14] proposed a Deep Learning method of using X-ray image of the lung to identify the presence of corona virus infection. Here, two concepts has been used such as Deep Neural Network (DNN) and Convolutional Neural Network $(\mathrm{CNN})$. The features of the image are extracted using the fractal technique and fed to DNN whereas direct lung image has been given as input to the CNN. After the experiment, it has been stated that the DNN has the efficiency of $83 \%$ where the CNN has the accuracy of $93 \%$.

Apart from using CT images, x-rays and ultrasound images, covid-19 can also be detected using clinical data from the patients [15]. These clinical data includes the age of the patient, symptoms of the patients, persistence of the symptoms, food intake of the patients etc. In this system, many algorithms such as logistic regression, SVM, Decision Trees, Naïve Bayes has been employed to process the clinical data of 212 suspected patients. This obtained an overall accuracy of around 95\%, however it may degrade with the increase of data. 


\section{System Architecture}

The following diagram depicts the architecture of the proposed system. At first, the CT scan input is pre- processed in order to remove any noise and the features of the image are extracted and given as input to the CNN. Simultaneously, clinical data from the patients are collected, processed for the information and given as another input to the CNN. By processing these two inputs, the CNN with the utilization of the trained data, provides the result as positive or negative. If the result is positive, then the system further refines the infection affected region.

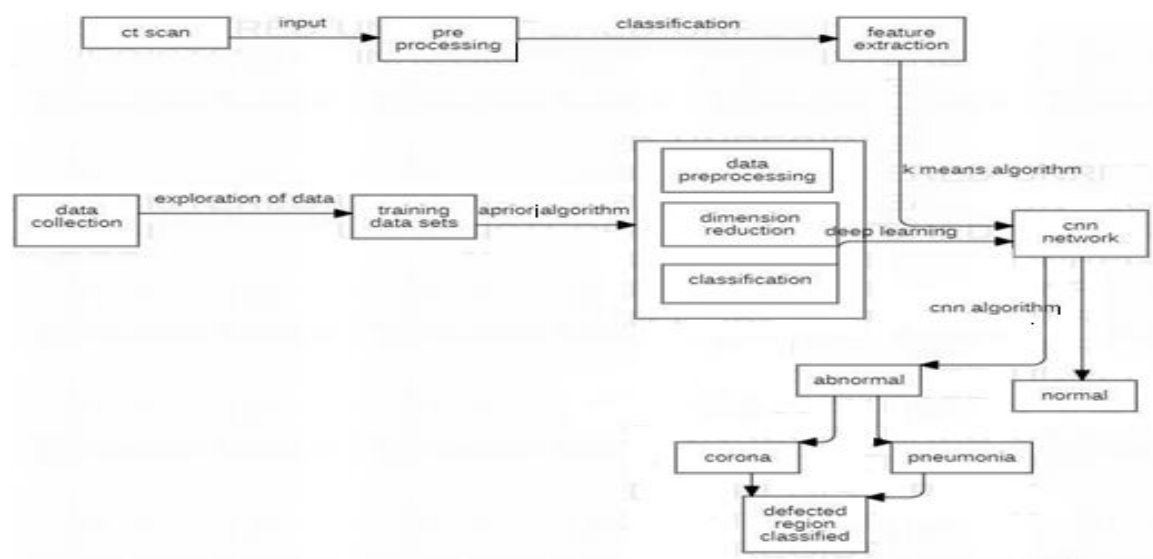

Figure 1. Architecture of the proposed system

\section{Data Flow Diagram}

\subsection{Data Flow Diagram 0}

Collect the datasets from the healthcare centres or from any reputational website. Preprocess the data to clear any distractions.

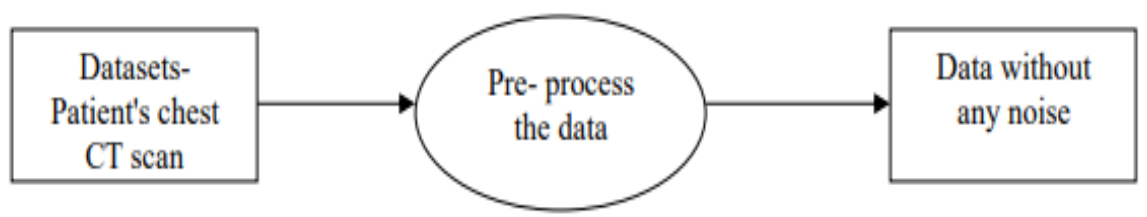

Figure 2. Data Flow diagram Level-0 


\subsection{Data Flow Diagram 1}

Characteristics are extracted from the pre-processed datasets and fed into the convolutional neural network. Simultaneously, physical data of the infected person is collected and trained and given as input to the CNN.

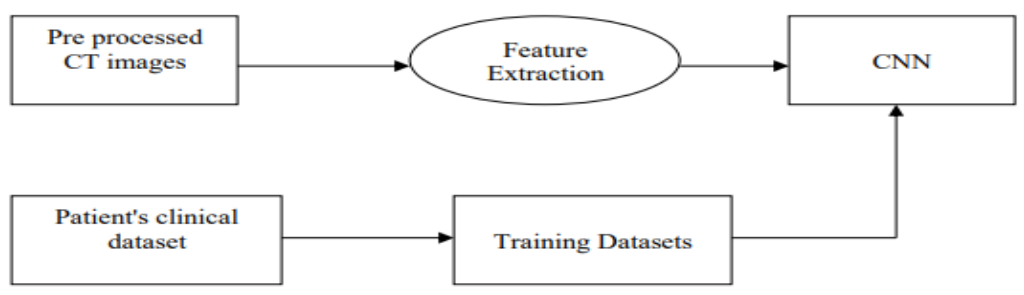

Figure 3. Data Flow Diagram Level-1

\section{Result And Discussion}

In the proposed system, we are using more than 6000 datasets for training and testing the CNN. When an input image along with the clinical data is given to the system, it produces the output with the efficiency if $97 \%$.

\section{Conclusion}

Since COVID-19 is an extraordinary pandemic that undermines the whole world and wellbeing laborers are attempting to distinguish the manifestations because of the shifted indications appeared by different tainted patients, the utilization of innovation can help them in managing the pandemic. It is seen that Artificial Intelligence assumes a significant part in different applications including the clinical field. All these AI based methods has its own constraints, for example, not separating the Coronavirus sore from pneumonia injuries, absence of more crown tainted patient's datasets, inferior quality of the accessible datasets, inaccessible of the clinical information of the patients and so on. All of these requirements must be vanquished or cleared so as to build the adequacy of conclusion of the disease.

\section{References}

[1] World Health Organization's announcement https:/www.who.int/emergencies/diseases/novelcoronavirus-2019/technical-guidance/naming-the-coronavirus-disease-(covid-2019)-and-the-virus-thatcauses-it

[2] https:/github.com/CSSEGISandData/COVID-19

[3] Livemint article - https:/www.livemint.com/science/news/coronavirus-likely-to-become-a-seasonaldisease-say-scientists-11600494712164.html

[4] COVID-19-Wikipedia https://en.wikipedia.org/wiki/Coronavirus_disease_2019

[5] CNET ARTICLE- https://www.cnet.com/health/coronavirus-testing-how-long-does-it-take-to-get-testresults-for-covid-19/

[6] MedicalNewsToday Article- https://www.medicalnewstoday.com/articles/coronavirus-causes 
[7] Worldometer report - https://www.worldometers.info/coronavirus/

[8] Vaccination Details- https://www.mohfw.gov.in/covid_vaccination/vaccination/index.html

[9] Indian Express Article - https://indianexpress.com/article/explained/bharat-biotechs-covaxin-hasaround-81-efficacy-what-does-this-mean-for-india-7212855/

[10] Swetha M. and Rajendiran M. - A Review on Effectiveness of Artificial Intelligence Techniques in the Detection of COVID-19/ DOI: 10.1109/SMART50582.2020.9336798.

[11] AnaïsScohy, AhalieyahAnantharajah, Monique Bodéus, Benoît Kabamba-Mukadi, Alexia Verroken, Hector Rodriguez-Villalobos," Low performance of rapid antigen detection test as frontline testing for COVID-19 diagnosis - Journal of Clinical virology, Volume 129, August 2020, 104455

[12] Natalia Budaa, Elena Segura-Graub, Jolanta Cylwikc, Marcin Wełnickid, Lung ultrasound in the diagnosis of COVID-19 infection - A case series and review of the literature. - Advances in Medical Sciences, Volume 65, Issue 2, September 2020, Pages 378-385

[13] Rodolfo M. Pereira, Diego Bertolini , Lucas O. Teixeira , Carlos N. Silla Jr. , Yandre M.G. Costa COVID-19 identification in chest X-ray images on flat and hierarchical classification scenarios / Volume 194, October 2020, 105532

[14] ShayanHassantabara, Mohsen Ahmadi b , Abbas Sharific , Diagnosis and detection of infected tissue of COVID-19 patients based on lung x-ray image using convolutional neural network approaches / Volume 140, November 2020, 110170

[15] AkibMohiUd Din Khanday Syed TanzeelRabani Qamar Rayees Khan Nusrat RoufMasaratMohiUd Din - Machine learning based approaches for detecting COVID-19 using clinical text data https://doi.org/10.1007/s41870-020-00495-9

[16] ShaopingHu,Yuan Gao, ZhangmingNiu, et al , Weakly Supervised Deep Learning for COVID-19 Infection Detection and Classification From CT Images, VOLUME 8, 2020, DOI 10.1109/ACCESS.2020.3005510.

[17] Xi Ouyang, JiayuHuoy, Liming Xia, et al, Dual-Sampling Attention Network for Diagnosis of COVID19 from Community Acquired Pneumonia", DOI 10.1109/TMI.2020.2995508, IEEE Transactions On Medical Imaging.

[18] Guotai Wang, Xinglong Liu, et al," A Noise-robust Framework for Automatic Segmentation of COVID-19 Pneumonia Lesions from CT Images , http://dx.doi.org/10.1109/TMI.2020.3000314

[19] Longxi Zhou, Zhongxiao Li, Juexiao Zhou, et al, A Rapid, Accurate and Machine-Agnostic Segmentation and Quantification Method for CT-Based COVID-19 Diagnosis ", DOI 10.1109/TMI.2020.3001810

[20] B.Akshaya, M.Rajendiran, “ Securing Documents Using Digital Certificate-A Review", International Journal of Research in Advent Technology (IJRAT), 2018

[21] HengyuanKangy, Liming Xiay, FuhuaYany, et al, Diagnosis of Coronavirus Disease 2019 (COVID19) with Structured Latent Multi-View Representation Learning",http://dx.doi.org/10.1109/TMI.2020.2992546

[22] Mohamed Abd Elaziz, Ahmed A. Ewees, et al , An Improved Marine Predators Algorithm With Fuzzy Entropy for Multi-Level Thresholding: Real World Example of COVID-19 CT Image Segmentation, DOI 10.1109/ACCESS.2020.3007928

[23] V.D.Ambeth Kumar, G.Gokul, S.Malathi, K.Vengatesan, D.Elangovan, B.Chitra, "Implementation Of The Pulse Rhythemic Rate For The Efficient Diagonising Of The Heart Beat", ”, Healthcare Technology Letters (IET) 2019 Apr 17;6(2):48-52.

[24] S.V. Ruphitha et.al, " Management of Major Postpartum Haemorrhage by using Zigbee protocol - A Review “, 2021 6th International Conference on Inventive Computation Technologies (ICICT) (DOI: 10.1109/ICICT50816.2021.9358757)

[25] Ambeth Kumar.V.D, Dr.M.Ramakrishnan, V.D.Ashok Kumar and Dr.S.Malathi (2015) "Performance Improvement using an Automation System for Recognition of Multiple Parametric Features based on Human Footprint" kuwait journal of science, Vol 42, No 1 (2015), pp:109-132.

[26] M.Ramakrishan et.al., "Footprint Based Recognition System" in the month of April for the International Journal Communication in Computer and Information System (CCIS) Journal (Springer) Volume 147, Part 3, 358-367, DOI: 10.1007/978-3-642-20573-6_63, April 2011

[27] Indhumathi.M et.al, "Healthcare Management of Major Cardiovascular Disease-A review", 2021 6th International Conference on Inventive Computation Technologies (ICICT), (DOI: 10.1109/ICICT50816.2021.9358519)

[28] Hema Kumar.S, J.Uday Kiran, V.D.AKumar, G.Saranya, Ramalakshmi V, "Effective Online Medical Appointment System", International Journal of Scientific \& Technology Research, Volume 8, Issue 09, September 2019, Pages $803-805$.

[29] Ambeth Kumar.V.D et.al .Novel Wireless Sensing System For The Welfare Of Sewer Laborers. Healthcare Technology Letters, Volume 5, Issue 4, pp. 107 -112, 2018 